\title{
Novel Models for the Extension Pneumatic Muscle Actuator Performances
}

\author{
Alaa Al-Ibadi \\ University of Salford \\ Computing, Science and Engineering \\ a.f.a.al-ibadi@edu.salford.ac.uk
}

\author{
Samia Nefti-Meziani \\ University of Salford \\ Computing, Science and Engineering \\ S.Nefti-Meziani@salford.ac.uk
}

\author{
Steve Davis \\ University of Salford \\ Computing, Science and Engineering \\ S.T.Davis@salford.ac.uk
}

\begin{abstract}
This paper illustrates the design, implementation and modelling of the extensor pneumatic muscle actuator (PMA). The extensor soft actuator has a vital feature of ability to bend and extend, and that give it the flexibility to use in numerous applications. The extended behaviour of this actuator is modelled mathematically to be used to predict the length of a wide range of actuators at different air pressure amounts and make the position control of such type of actuator easier and precise. Moreover, the contraction force formula is modified to describe the pushing force for the extensor actuator. The bending behaviour of single muscle is explained and a 4-PMA continuum arm has been constructed to study its performance and model the bending angle.
\end{abstract}

Keywords- Extensor Pneumatic Muscle Actuator; Performances; Mathematical Models

\section{Introduction}

Soft actuators have grown formidable reputation among researchers, predominantly in the last few decades. As a result, soft robotics has been extensive use in the numerous areas. Researchers from different fields such as material science, physics, biology and computer and control engineering interest in such type of robots [1]. This developing research field emphases on robots made of soft materials which increase the safety of human-robot interaction, and make the robot more compliance to its environment [2], [3]. An infinite number of freedoms (DOF) are achievable by soft robots due to the bending capability of the soft actuator and as a result, a robot end effector can reach every point in the 3D workspace [4]. Soft robots have a further positive over rigid robots, where they produce slight resistance to obstacles and can adapt to them. Consequently, soft and fragile payloads could be handled without causing any damages [5].

A pneumatic muscle actuator (PMA) which is presented by Joseph L. McKibben in the 1950's, is built from an inner rubber tube bounded by a braided sleeve [6] has been used to create soft robots. The construction of the PMA defines the type of actuator, by selecting the length of both the inner tube and the braided sleeve the PMA act as a contraction actuator if the braided angle is less than $54.7^{\circ}$, and the extension actuator if the angle is more than $54.7^{0}$. Moreover, the PMA will produce a pulling force for contractor type and a pushing force for the extensor one.

Important researches have been done to define the contraction force of the pneumatic muscle actuators. Among these researches, the Chou and Hannaford model
[6] and Tondu and Lopez model [7] are commonly referred.

In this paper, different lengths of an extensor PMAs have been constructed to study their performance and we have written a set of mathematical equations to describe the change of length with pressure input. In addition, enhancement and modification have been done to the contraction force formula to fit the extension behaviour. Moreover, a 4-PMA extensor continuum arm is built and the bending angle at different attached load is formulated.

\section{EXTENSION PMA}

The construction of the PMA defines its action as a contraction or extension actuator by controlling the braided angle $(\theta)$; as identified in the force formula below, the force either be pulling or pushing depends on $(\theta)$. A photograph of a $30 \mathrm{~cm}$ extensor PMA is shown in Fig.1. Generally, the PMA is constructed from an inner rubber tube which is covered by a braided sleeve with two solid material terminals which are fixed strongly to ensure that no air leakage occurs. One of the terminals has a small hole for input and output of actuated air. Fig.2 shows a basic diagram of PMA with "L" length, "D" diameter and " $\theta$ " angle between the vertical axis and braided strand $(\mathrm{b})$.

When the length of the braided sleeve is more than the length of the rubber inner tube in sufficient amount, the braided angle $(\theta)$ is more than $54.7^{\circ}[8]$, in this project the braided sleeve length is 2.8 times of the inner tube. The percentage of extension differs from one muscle to another, and it is not less than 50\% [9].

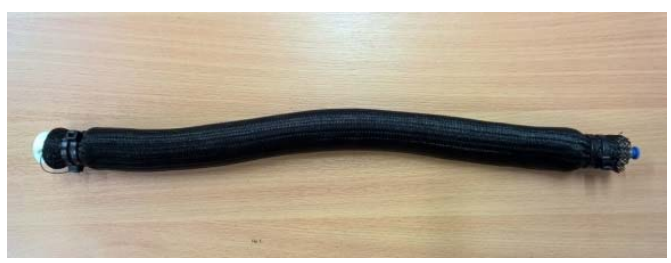

Figure.1 The photograph of the $32 \mathrm{~cm}$ extensor PMA

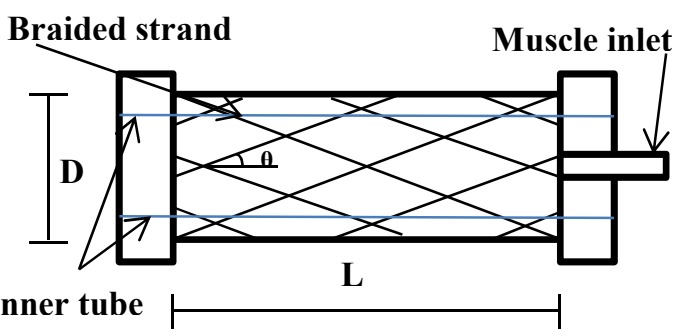

Figure.2 Pneumatic muscle actuator diagram 


\section{A MATHEMATICAL MODEL FOR EXTENSOR LENGTH}

The sigmoidal shape of muscle length as is shown in Fig.3, leads to modelling the length of PMA performance as a mathematical sigmoid function. To model the length with input air pressure, three muscles are used for initial lengths " $\mathrm{L}_{0}$ " $(20,30 \& 40 \mathrm{~cm})$, we chose these lengths to cover a range of muscles lengths, and then select another PMA's dimensions to validate our model. All these Muscles have the same initial diameter of $(3.3 \mathrm{~cm})$ and an initial braided angle of $\left(71.5^{\circ}\right)$. We have considered each muscle and recorded how its length has changed with gauge pressure "p". Fig. 3 shows the length of the PMAs and it is clear that there is a significant matching between experiment and model plots for different set of data. From the first set of the pneumatic muscles data, we define a set of equations depending on " $\mathrm{L}_{0}$ and p". Equation (1) describes the length of the pneumatic muscle with both $\left(\mathrm{p} \& \mathrm{~L}_{0}\right)$, and (2) gives the parameter values of (1), which it depends on the initial length of the PMA $\left(\mathrm{L}_{0}\right)$.

$$
\begin{gathered}
L=a-\frac{b}{1+\left(\frac{p}{c}\right)^{d}}+0.0009 L_{0} p^{2} \\
\text { Where: }\left[\begin{array}{l}
a \\
b \\
c \\
d
\end{array}\right]=\left[\begin{array}{ccc}
0.2281 & 0.084598 & -0.0013189 \\
0.94047 & 0.095934 & -0.0014863 \\
0.014934 & 0.0012083 & -0.000026277 \\
0.2080174 & -0.0032043 & 0.0000106189
\end{array}\right]\left[\begin{array}{l}
L_{0} \\
L_{0}^{2} \\
L_{0}^{3}
\end{array}\right]
\end{gathered}
$$

The mathematical model of the extensor actuator is formulated to fit the muscle lengths from $20 \mathrm{~cm}$ to $40 \mathrm{~cm}$ under the specifications list in table 1 at no-load.

Table.1. The initial specifications of the three PMAs

\begin{tabular}{|c|c|c|c|c|}
\hline $\mathrm{L}_{0}(\mathrm{~m})$ & $\begin{array}{c}\text { Rubber } \\
\text { thickness }(\mathrm{m})\end{array}$ & $\begin{array}{c}\text { Braided } \\
\text { thickness (m) }\end{array}$ & $\begin{array}{c}\text { Inner } \\
\text { diameter }(\mathrm{m})\end{array}$ & $\begin{array}{c}\text { Rubber } \\
\text { stiffness }(\mathrm{N} / \mathrm{m})\end{array}$ \\
\hline 0.2 & $1.1 * 10^{-3}$ & $0.5 * 10^{-3}$ & $12^{*} 10^{-3}$ & 363.33 \\
\hline 0.3 & $1.1 * 10^{-3}$ & $0.5 * 10^{-3}$ & $12^{-3} 10^{-3}$ & 363.33 \\
\hline 0.4 & $1.1 * 10^{-3}$ & $0.5 * 10^{-3}$ & $12^{*} 10^{-3}$ & 363.33 \\
\hline
\end{tabular}

To validate this formula another actuator has been constructed for the same specifications but with $25 \mathrm{~cm}$ initial length.

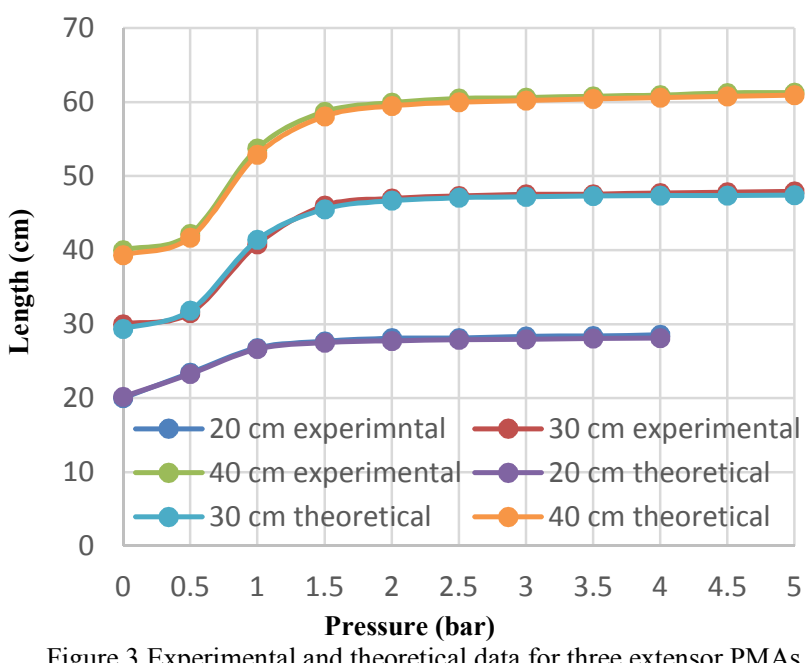

Fig.4 illustrates both the experimental and model length characteristics for the new muscle, which it proved the validity of the extensor length formula in (1) and (2).

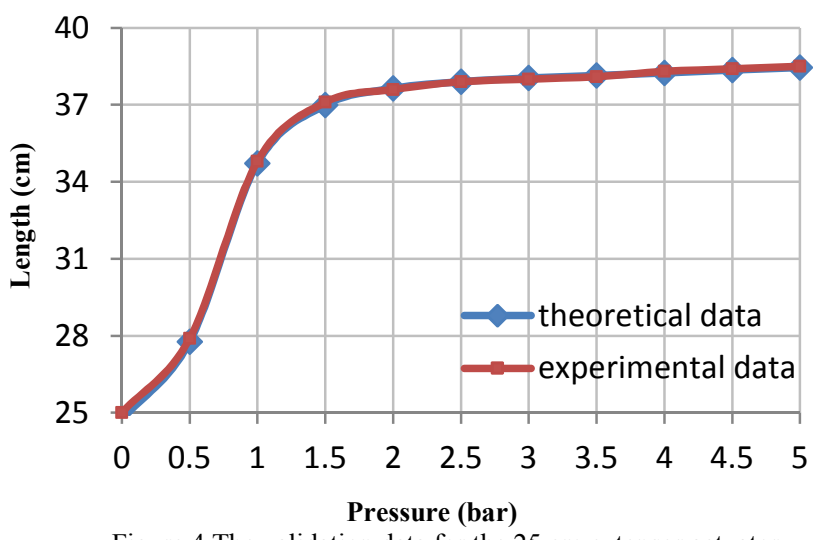

Figure. 4 The validation data for the $25 \mathrm{~cm}$ extensor actuator.

\section{MODELling OF PUSHING ForCE}

The muscle actuator converts the air energy into mechanical form by transferring the pressure input to the muscle into the extensor force. Referring to the virtual work theory, the varying of input work $\left(\mathrm{w}_{\mathrm{in}}\right)$ of the pneumatic muscle is:

$$
d w_{\text {in }}=p \cdot d v
$$

Where $(d v)$ is the volume change of the actuator. The output work ( $\mathrm{w}_{\text {out }}$ ) changes with change of length, as in the following equation:

$$
d w_{\text {out }}=f . d L
$$

Fig. 5 shows the relation between the parameters of the PMAs. Equations (5), (6) and (7) define the Tondu and Lopez force model under the following assumptions: 1- The shape of the PMA is a perfect cylinder with zero wall thickness. 2- There is a contact between the inner rubber tube and the braided sleeve. 3- The braided strand length is constant. 4- There is no friction between the tube and the sleeve. 5- Neglecting the latex tube force [7].

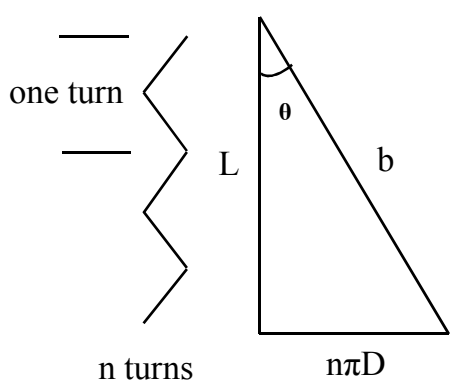

Figure.5 Parameters description of PMA

Where from fig.5:

$$
L=b \cos \theta, D=b \frac{\sin \theta}{n \pi}
$$


By considering (5) the strand length can be evaluated as:

$$
b=\left(L^{2}+(D n \pi)^{2}\right)^{\frac{1}{2}}
$$

The force "f" can be calculated as the multiplication of the gauge pressure and the volume change with respect to length.

$$
f=-\pi r_{0}^{2} p\left[\alpha(1-\varepsilon)^{2}-\beta\right]
$$

Where: $\varepsilon=\frac{L-L_{0}}{L_{0}}, \alpha=\frac{3}{\tan ^{2}\left(\theta_{0}\right)}$, and $\beta=\frac{1}{\sin ^{2}\left(\theta_{0}\right)}$.

Moreover, " $r_{0}$ and $\theta_{0}$ " represent the initial values of radius and angle of the PMA respectively; and " $\varepsilon$ " is the extension ratio. Fig. 6 below shows the experimental force data with the plot of (7) for the $30 \mathrm{~cm}$ PMA. This figure illustrates that the difference between the theoretical and experimental records has two main causes. The first one is the non-cylindrical shape of the PMA at zero or low air pressure supplied. The second reason is that there is no extremely contact between the inner rubber tube and the braided sleeve and the resistance of rubber tube. To overcome these two aspects, the correction factor " $q(p)$ " is used by multiplying it by the extension ratio of (7).

$$
q(p)=-\left(1+e^{-0.5 p}\right)
$$

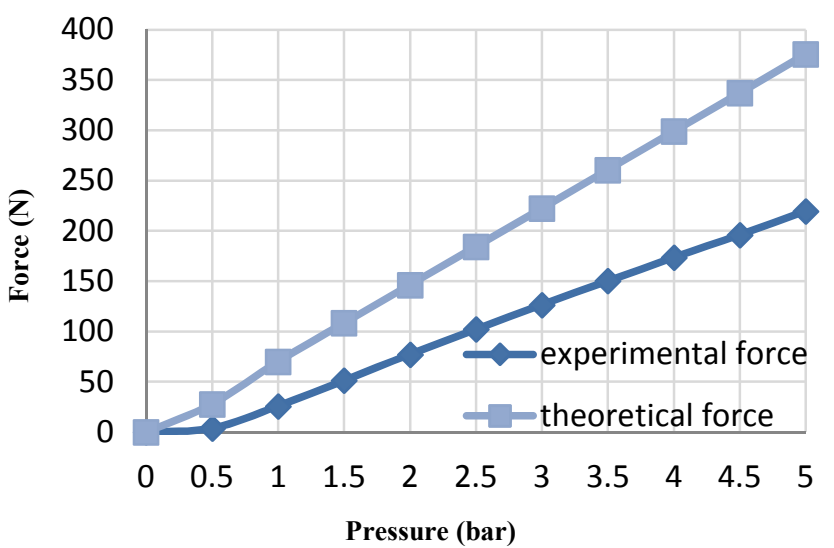

Figure.6 The experimental and the theoretical [18] force for the 32 cm extensor PMA

The increasing in gauge pressure caused an increasing in the correction factor, therefore, at high pressure, the shape of the PMA becomes perfectly cylindrical. On the other hand, the experimental data shows that the amount of useless (zero force) pressure is $(0.45$ bar). Due to the resistance of inner tube against the stretch and the contact less between the braided sleeve and the inner tube, the energy of actuated air below 0.45 bar will be considered a loses, for that the extensor force in (7) is defined to be $(0 \mathrm{~N})$ from $(0-0.45$ bar $)$ and the gauge pressure shift by $(0.45$ bar $)$ otherwise. The zero force pressure is studied by Tsagarakis and Caldwell [10] as a function of pressure and inner tube diameter. Here, it is found that this constant value could be considered for this type of PMAs.

Taking these two factors into account gives substantial matching between the experimental and theoretical force characteristics. The force of all actuators under study has been validated and Fig. 7 gives the force plot for the $(30 \mathrm{~cm})$ PMA.

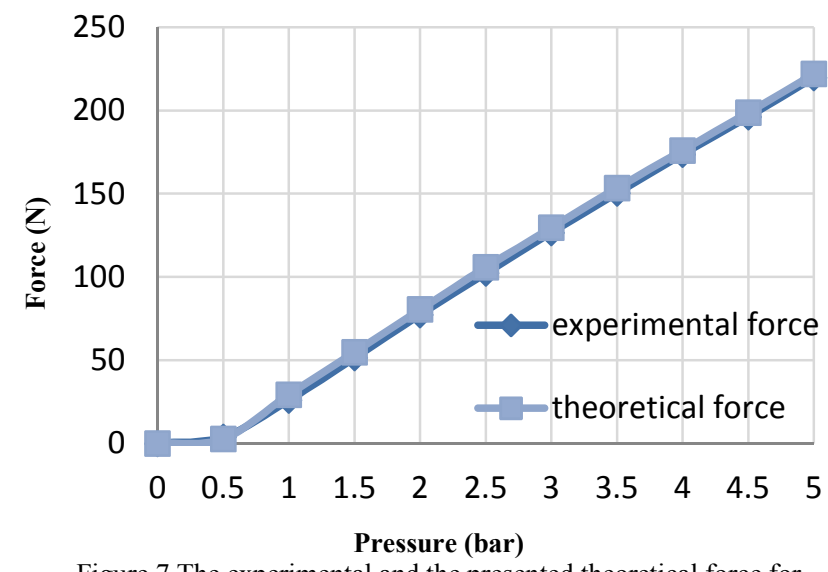

Figure.7 The experimental and the presented theoretical force for the $30 \mathrm{~cm}$ extensor PMA

The modification of (7) shows in (9) below:

$$
\begin{gathered}
f=0, \quad 0 \leq p \leq 0.45 \text { bar } \\
f=-\pi r_{o}^{2}(p-0.45)\left[\alpha(1-q \varepsilon)^{2}-\beta\right], p \geq 0.45
\end{gathered}
$$

\section{LOAD EFFECTS ON ACTUATOR BEHAVIOUR}

Attaching load to the extensor PMA changes its extension ratio. Fig. 8 shows that change with respect to attached load at gauge pressure (1-5) bar. Increasing the attached load gives the PMA more ability to extend at low pressure (p). In this figure, all lines intersect around (3.5) $\mathrm{Kg}$.

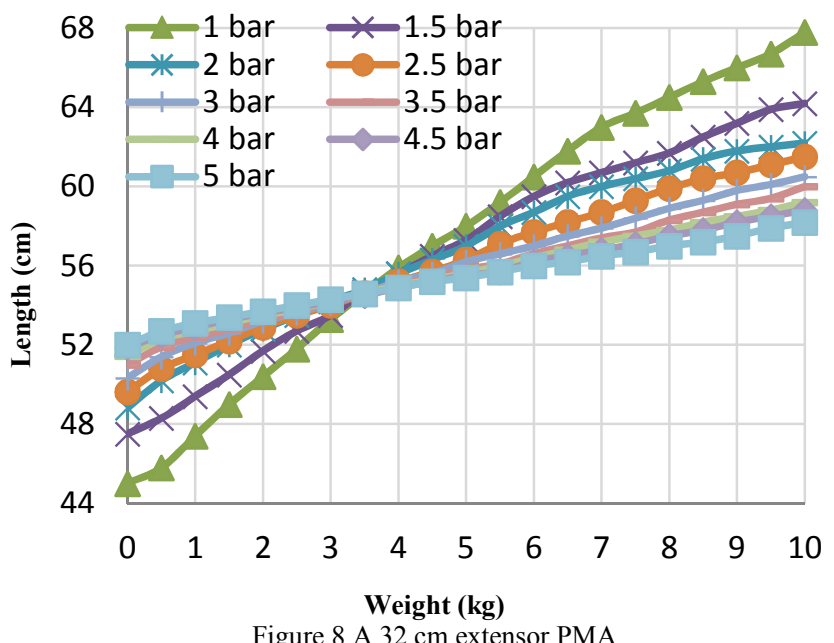

Figure. 8 A $32 \mathrm{~cm}$ extensor PMA 
The load test is done as follows: 1- Attached different loads up to $10 \mathrm{Kg}$ in $0.5 \mathrm{Kg}$ steps at 1 bar. 2Increase the pressure to 5 bar in 0.5 bar steps and repeat step (1) each time. Fig.9 shows that the behaviour of the muscle is changed after load $3.5 \mathrm{Kg}$, where it is clear that increasing the gauge pressure (p) makes the PMA act as a contraction muscle instead of an extension muscle.

From Fig.8 and Fig.9, the extensor muscle might be used as a contractor actuator when it is loaded with more than $3.5 \mathrm{~kg}$.

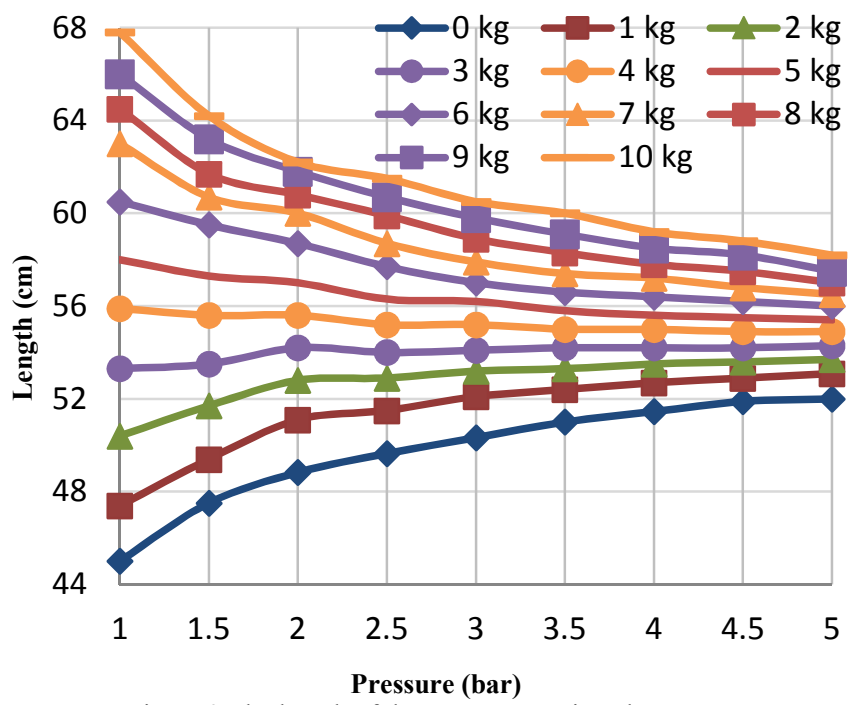

Figure.9 The length of the actuators against the pressure at fixed weight values

As mentioned in section 2 above, when the braided angle is more than $54.7^{0}$, the actuator acts as an extensor muscle. On the other hand, it works as a contractor muscle when $(\theta)$ is less than $54.7^{\circ}$. The high angle occurred as a result of the long braided sleeve compared with the inner tube. In this paper (2.8 times) of braided sleeve longer than the inner tube has been used. The elasticity characteristic of the rubber tube gives the muscle a capability of elongation under the load effect, making $(\theta)$ less than the threshold value. As a result, the actuator behaviour changes to contraction. This performance provides an additional advantage for the extensor PMA and makes it able to extend first at a certain pressure at no load then carries a certain weight then pick up it at high pressure.

\section{EXTENSOR CONTINUUM ARM}

By fixing one side of the extensor muscle and prevent it to extend at pressurised condition, the actuator will bend related to the air pressure [11] as shown in Fig.10. In this figure a thread is used to fix the length of the actuator longitudinally.

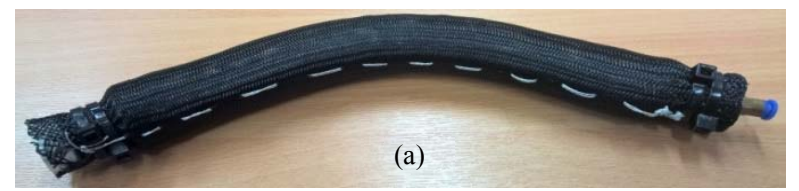

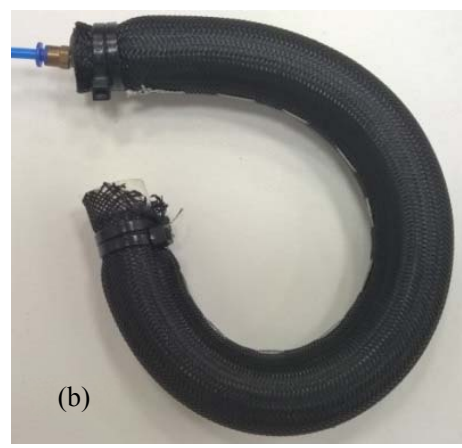

Figure.10 A $30 \mathrm{~cm}$ extensor PMA (a) one side sewed actuator (b) bending under 3 bar air pressure.

To develop the bending in all directions a 4-PMAs continuum arm is designed and constructed as shown in Fig. 11 and Fig.12. Four extension actuators $30 \mathrm{~cm}$ each are used; one in the centre and the others are located at 3 $\mathrm{cm}$ from the centre and $120^{\circ}$ between each other.

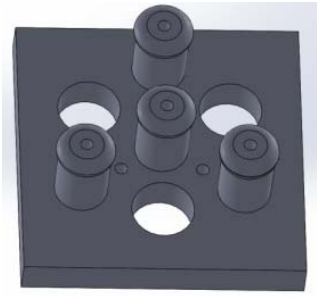
(a) Fixed end Figure.11 So

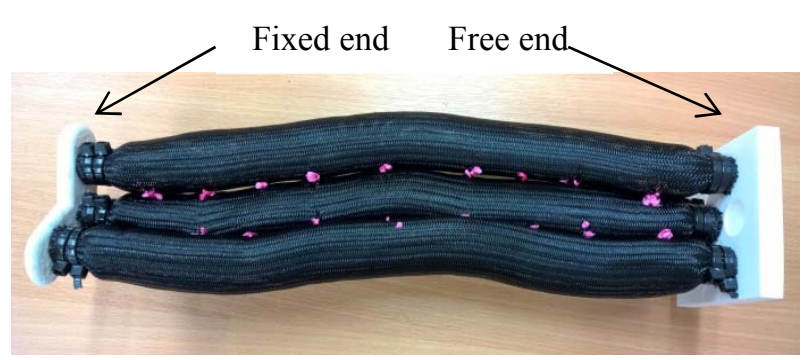

Figure.12 Four $30 \mathrm{~cm}$ extensor PMAs continuum arm

A Solidworks 2015 is used to design the two ends, and a $3 \mathrm{D}$ printer is used to print them. Then the centre actuator is connected to others individually. Equal air pressure in all PMAs makes the arm extend in a straight direction, while, the different pressure inside the four actuators make the free end move in all direction in the space at almost constant curvature [11]. The position angle is observed as a function of the supplied pressure and the attached load.

Firstly, the experiments are done by recording the initial angle of the free end $(\delta)$, which it is equal to (zero) degree due to a straight-line arm. Secondly, all PMAs are actuated by ( 0.45 bar $)$, then the pressure is increased in one of the PMAs in the corner. The arm will then bend into another position, depending on the amount of the pressure in the muscle. And $\delta$ is recorded each time. Fig. 13 shows the contraction arm under actuation from certain pressure between $0-5$ bar. 
The maximum angle value ( $\delta$ max) depends on the amount of the attached load (w) to the arm end. Table 2 below shows different maximum angles with different load values.

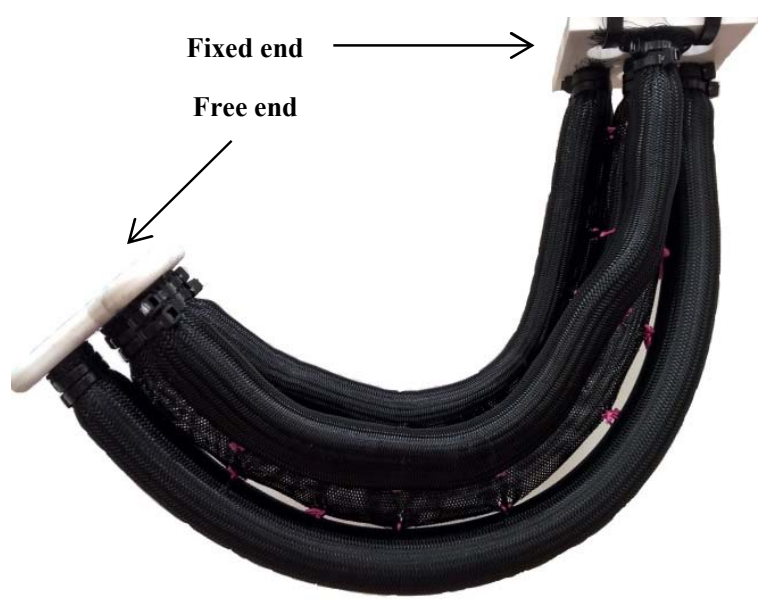

Figure.13 An extensor continuum arm at certain pressure

Table. 2 maximum angle with different loads

\begin{tabular}{|c|c|c|}
\hline Load w (Kg) $_{\text {(Kg }}$ & Pressure P (bar) & $\boldsymbol{\delta}_{\max }$ (degree) \\
\hline 0.0 & 5.0 & 164.833 \\
\hline 0.1 & 5.2 & 163 \\
\hline 0.2 & 5.0 & 155 \\
\hline 0.3 & 5.2 & 135.2 \\
\hline 0.4 & 5.0 & 126.1 \\
\hline 0.5 & 5.0 & 116.2 \\
\hline
\end{tabular}

The bending angle against the air pressure at different load values is illustrated in Fig. 14 which it represents the position of the free end at any pressure step.

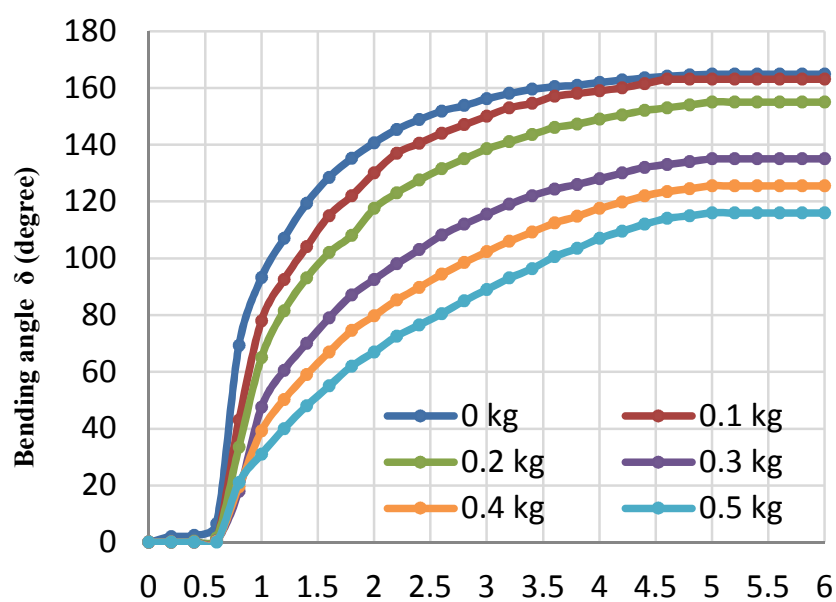

icsourc (vas)

Figure.14 The bending angle against the pressure at different load conditions
From these data, the bending angle increase when the applied pressure increase and its value at each air pressure step depends on the attached load. In our experiment, various loads from 0 to $0.5 \mathrm{~kg}$ are attached. Furthermore, the arm starts to bend when the air pressure reaches 0.8 bar and the angle has a fixed value $(\delta \max )$ over 5.2 bar. As a result, the operation range will be at pressure values from 0.8 bar to 5.2 bar.

A new formula of bending angle is presented as a function of the input air pressure and the amount of attached load as following:

$$
\delta=\mathrm{a}-\frac{\mathrm{b}}{\left[1+\left(\frac{\mathrm{p}}{\mathrm{c}}\right)^{\mathrm{d}}\right]^{\mathrm{e}}}
$$

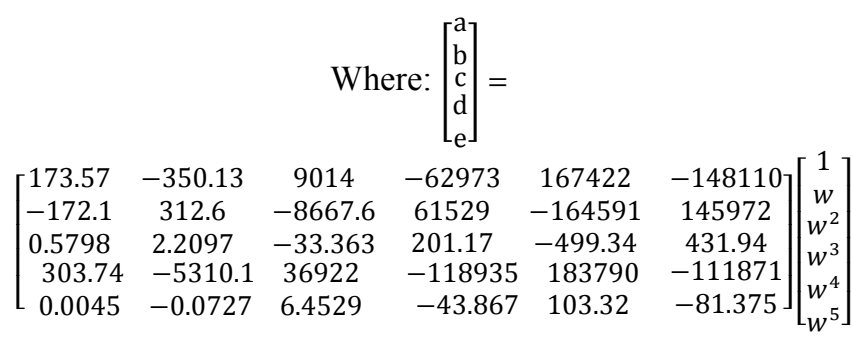

Equation (10) above gives the bending angle at any air pressure amount from $0-6$ bar, while, the value of the parameters $(a, b, c, d$ and e) depends on the attached load.

This formula is validated for all load conditions and Fig. 15 gives the validation results for three conditions $(0$ $\mathrm{kg}, 0.2 \mathrm{~kg}$ and $0.5 \mathrm{~kg}$ ). This figure shows a significant matching between the experimental results and the presented formula.

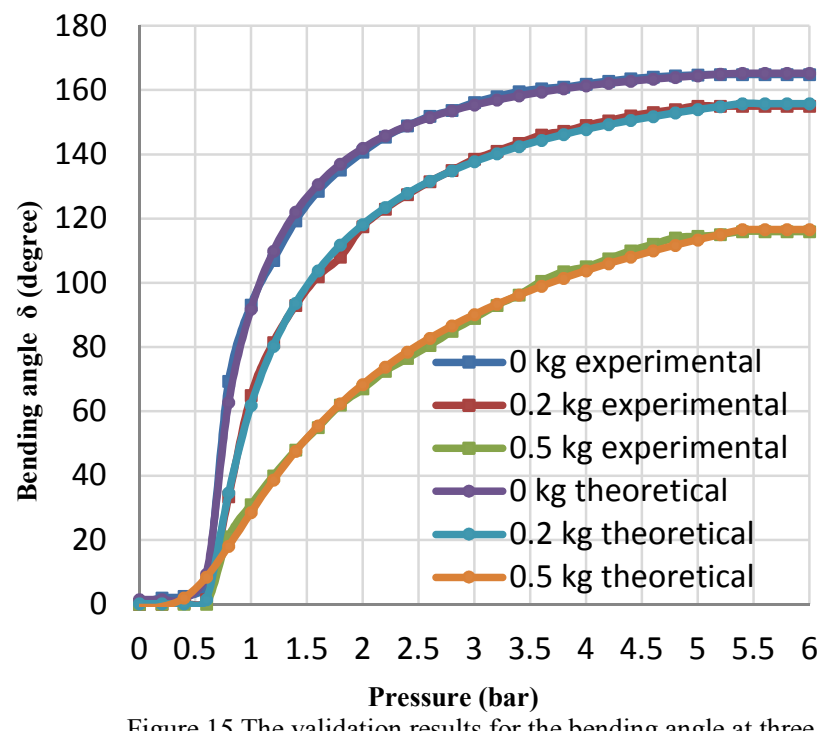

Figure.15 The validation results for the bending angle at three different load conditions 


\section{CONCLUSION}

An experimental model for the length of an extensor pneumatic muscle actuator is presented in this paper by two equations. These formulas show minimum error between the model and experimental data for muscles with any nominal length $\mathrm{L}_{0}$ between $20 \mathrm{~cm}$ and $40 \mathrm{~cm}$. On the other hand, calculating the specific zero force pressure in addition to using the shape correction factor make the force model formula a more accurate and substantial match with the experimental data for all muscle lengths under study.

The experimental data gives the capability to use the extensor actuator as a contractor at a specifically attached load. This ability makes this type of PMA appropriate for multi purposes.

An extensor arm is built from four extensor PMAs and we studied its behaviour for bending. The experiments show high bending angle at load up to 0.5 $\mathrm{kg}$. A formula for this bending angle is presented for noload and load conditions. The validation of this formula shows a significant accuracy for the air pressure more than 0.8 bar.

As a future work, the three dimension positions for the free end can be tested and modelled a function of air pressure in each actuator to make the position control of continuum arms more efficient.

\section{ACKNOWLEDGMENT}

The authors would like to thank the ministry of higher education/Iraq, University of Basrah, computerengineering department for providing scholarship support to the first author of this paper.

\section{References}

[1] L. Margheri and B. Trimmer, "Soft Robotics Community Events: Meeting Different Backgrounds for Common Challenges," Soft Robotics, vol. 1, pp. 236-238, 2014.

[2] R. Mutlu, S. K. Yildiz, G. Alici, M. in het Panhuis, and G. M. Spinks, "Mechanical stiffness augmentation of a 3D printed soft prosthetic finger," in Advanced Intelligent Mechatronics (AIM), 2016 IEEE International Conference on, 2016, pp. 7-12.

[3] T. E. Pillsbury, Q. Guan, and N. M. Wereley, "Comparison of contractile and extensile pneumatic artificial muscles," in Advanced Intelligent Mechatronics (AIM), 2016 IEEE International Conference on, 2016, pp. 94-99.

[4] D. Trivedi, C. D. Rahn, W. M. Kier, and I. D. Walker, "Soft robotics: Biological inspiration, state of the art, and future research," Applied Bionics and Biomechanics, vol. 5, pp. 99-117, 2008.
B. A. Jones and I. D. Walker, "Kinematics for multisection continuum robots," IEEE Transactions on Robotics, vol. 22, pp. 43-55, 2006.

[6] C.-P. Chou and B. Hannaford, "Measurement and modeling of McKibben pneumatic artificial muscles," IEEE Transactions on Robotics and Automation., vol. 12, pp. 90-102, 1996.

[7] B. Tondu and P. Lopez, "Modeling and control of McKibben artificial muscle robot actuators," Control Systems, IEEE, vol. 20, pp. 15-38, 2000.

[8] W. Liu and C. Rahn, "Fiber-reinforced membrane models of McKibben actuators," Journal of Applied Mechanics, vol. 70, pp. 853859, 2003.

[9] W. McMahan, V. Chitrakaran, M. Csencsits, D. Dawson, I. D. Walker, B. A. Jones, et al., "Field trials and testing of the OctArm continuum manipulator," in Robotics and Automation, 2006. ICRA 2006. Proceedings 2006 IEEE International Conference on, 2006, pp. 23362341.

[10] N. Tsagarakis and D. G. Caldwell, "Improved modelling and assessment of pneumatic muscle actuators," in IEEE International Conference on Robotics and Automation, 2000. Proceedings. ICRA'00. , 2000, pp. 3641-3646.

[11] I. D. Walker, "Continuous backbone "continuum" robot manipulators," ISRN Robotics, vol. 2013, 2013. 